\title{
Optimal generator matrix G
}

\begin{abstract}
Multiple antenna transmission methods are currently being developed all around the world for evolving 3G wireless standards. Space-Time Trellis Code (STTC) has been proven to use transmit diversity efficiently. It effectively exploits the effects of multipath fading to increase the information capacity of the multiple antenna transmission systems. STTC is a channel coding technique that maximises the 'distance' between different symbol matrices such that the probability of transmission errors are decreased when transmitting redundant symbol or in other words, to maximise the minimum determinant. Maximising the minimum determinant is equivalent to obtaining optimal generator matrix G. Instead of using state diagrams, optimal generator matrix $\mathrm{G}$ discussed in this paper is obtained using an improved algorithm which is based on Lisya tree structure. Optimal generator matrix $G$ in this paper has a minimum determinant of 48 which is the highest coding gain obtained so far.
\end{abstract}

Keyword: Space-time trellis code; Minimum determinant; Generator matrix; Coding gain; Pairwise error probability 